\title{
LINGUISTICS
}

\section{Emotive Verbs in English Sentiment Lexicons}

\author{
O. Baranovska \\ Ivan Franko National University of Lviv, Lviv, Ukraine \\ Corresponding author. E-mail: baranovska8719@gmail.com \\ Paper received 26.05.20; Accepted for publication 10.06.20.
}

\section{https://doi.org/10.31174/SEND-HS2020-230VIII38-01}

\begin{abstract}
The paper focuses on the representation of verbs in the existing sentiment lexicons of English. Sentiment lexicons are indispensable parts of sentiment analysis electronic tools. Though they contain a relatively small number of verbs in comparison with nouns or adjectives, still the occurrence of emotionally charged predicates in speech is a powerful sentiment marker. Thus, it is worthwhile comparing the verbs in the lexicons in order to discover the similarities and divergences across the lexicons and estimate the resources' performance based on the 'quality' of their verb content.
\end{abstract}

Keywords: sentiment lexicon, sentiment (emotive) verbs, sentiment analysis, polarity, connotation.

1. Introduction. The problem of identifying emotive lexis and building sentiment lexicons has a long history and can be tackled in various ways. Emotions 'foreign to the word of language' is a deep-rooted view in linguistics, emphasizing the need for context to identify emotionally charged items: "[...] emotion, evaluation and expression are foreign to the word of language and are born only in the process of its live usage in a concrete utterance [...]. They acquire their expressive coloring only in the utterance" [3, p.87].

Still, a number of scholars claim that there exist certain formal features that allow outlining emotive vocabulary, e. g., emotive affixes, reference to specific registers, etc. [17]. For instance, English prefix be- (becloud, bedevil, bedew, bedrop, befeather, beflower, etc.), conversion (to ape, to peacock, to hawk, to claw, etc), blending (guesstimate, recomember, phub), phrasal verbs (bear out, beat into, beef about, beat up, beg off, etc), low register verbs according to this view are the formal markers of potential emotive verbs.

Modern cognitive linguistics acknowledges the presence of emotions in language: "Reviewing theories of emotion and the course of language acquisition, it is argued that affect expression and affect conceptualization are both present in language..." [9, p.135]. Hence, it is worthwhile looking for emotive clues in language.

It was not until the first acclaimed corpora appeared that the study of usage proved to be particularly beneficial for the compilation of dictionaries, developing language materials that approximate the model of a natural language system. Processing vast linguistic material extracted from corpora contributed to the study of once unattainable connotation, presumably a carrier of emotions. A promising direction in recent corpus linguistics is the study of semantic prosody (or discourse prosody), i.e., a kind of connotative coloring of a lexical item resulting from a given word taking on the affective meaning common to all its typical collocates $[12 ; 18 ; 19]$. According to John Sinclair, semantic prosody is an obligatory component of the extended meaning of any lexical item [18]. The primary function of semantic prosody is to express attitude or evaluation [12,p. 58]. Accordingly, they distinguish positive and negative semantic prosody based on corpus collocations. For instance, cause has a negative semantic prosody due to its prevailing negative collocates: to cause trouble/ a problem/ chaos/ a mess/ misunderstanding/ an accident, etc.
In vein with the recent findings in corpus linguistics is the study of text sentiment. Sentiment analysis is a field of study that analyzes people's opinions, sentiments, evaluations, appraisals, attitudes, and emotions towards entities such as products, services, organizations, individuals, issues, events, topics, and their attributes $[11,5]$. Nowadays it has become a computational study based on probability models. An essential part of sentiment analysis, which in the long run determines its success, is the application of special sentiment lexicons. The compilation of sentiment lexicons became possible due to interdisciplinary study of sentiment and emotions encompassing traditional lexicography, corpus linguistics, psycholinguistics, computational linguistics, etc. Currently, the most attested electronic sentiment lexicons are SentiWordNet 3.0, Harvard General Inquirer, LIWC, MPQA Subjectivity Lexicon, and Bing Liu's Opinion Lexicon. Whether based on introspection and experiments, or corpus-driven data, or trying to implement both approaches, the lexicons require comparison in terms of their coverage and performance when applied for sentiment analysis. The attempts to evaluate the resources have been undertaken previously [1]. A special sentiment symposium tutorial and demo versions by Christopher Potts [15] have been created in order to visualize the lexicons' similarities and discrepancies and identify the best praxis of using sentiment lexicons effectively (http://sentiment.christopherpotts.net/lexicons.html). The lexicons mostly differ in terms of coverage, methods of creation, lexical units and their granularity. They are regularly enhanced and updated, new versions with higher performance are released. Sentiment lexicons as hands-on tools for automated opinion mining belong to the fundamental prerequisites of a successful text processing in terms of identifying affect, bias, attitude, emotions. Items belonging to any part of speech can potentially enter sentiment lexicons. Nonetheless, the existing sentiment lexicons are based on lexical parts of speech: nouns, adjectives, verbs and adverbs.

The aim of this paper is to study the distribution of English verbs across the available sentiment lexicons. Verbs as sentiment (emotion) carrying items are normally unduly overshadowed by the study of other word classes, in particular nouns and adjectives. To the best of my knowledge, verbs as part of sentiment lexicon have not 
been sufficiently studied so far.

2. Existing Sentiment Lexicons. Sentiment lexicons are generally defined as dictionaries of words with labels specifying their sentiments compiled to assist in identifying the sentiment of text. The sentiment may be represented in several possible forms, some of which are:

1. Fixed categorization into positive or negative,

2. A finite number of graded sets such as strongly positive, mildly positive, neutral, mildly negative, strongly negative,

3. A real value denoting sentiment strength in an interval such as $[-1 ;+1][1]$.

There are two broad approaches to the creation of sentiment lexicons - manual and automated. The major advantage of the manual approach is that since the annotation is performed by humans, correctness is guaranteed barring an actual error in annotation. The problem with this approach, however, is the immense time investment required.

While there are several methods to create automated sentiment lexicons, one of the most popular is to create a set of starting seed words with known sentiment orientation, and then expand that seed set usually with synonyms using an already existing lexical resource. The advantages of the automated approach in terms of the promise of high coverage are achieved only by a trade-off in accuracy. Annotating expressions of opinions and emotions in language has become a topical issue [21].

The following section discusses the methods of creation of each of the sentiment lexicons under scrutiny in this paper.

2.1. SentiWordNet as an automated sentiment lexicon

One way of creating a sentiment lexicon based on WordNet synsets and their associated sentiment is to iterate over WordNet synsets and assign sentiment scores to these synsets by means of a classifer which analyzes the glosses associated with the individual synsets. An example of a sentiment lexicon thus generated is SentiWordNet [4], where eight classifiers have been used to analyze the gloss of each WordNet synset in order to assign scores quantifying how objective Obj(_), positive Pos(_), and negative $\mathrm{Neg}\left(\_\right)$each synset is. Each score is determined by the (normalized) proportion of the eight classifers that have assigned the corresponding label to it and the sum of the three scores is constrained to equal 1 for each synset. The objectivity, positivity, and negativity scores for all 117,659 WordNet synsets have been computed in such a way. The scores thus obtained have been evaluated on a set of 1,105 WordNet synsets which have been scored in a similar fashion by five human annotators.
The peculiarities of SentiWordNet values are as follows:

1. The sentiment is tied intimately to the meaning of a word rather than the word itself.

2. A synset is can be either positive or negative, or neither positive nor negative (neutral, objective).

3. The scalar sentiment evaluation.

Figure 1 features a SentiWordNet visualization triangle WordNet synsets polarity: the red colour stands for the negative polarity, the green one for the positive one and the blue one for the neutral.

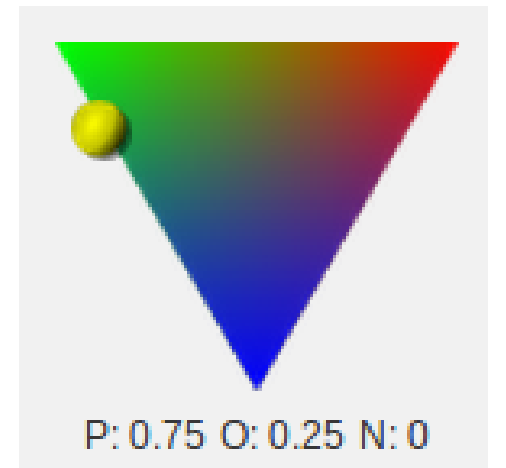

Figure 1. Visualization of synset beautiful\#1 in SentiWordNet (Adopted from Ahire 2015)[1]

Table 1 presents a summary of the sentiment of all SentiWordNet categories (parts of speech). As it can be inferred from the table, the vast majority of verbs have objective meaning (0.940). Negative verbs (0.034) somewhat prevail over the positive ones (0.026). Curiously enough, there are more positive sentiment verbs $(0.026)$ than positive sentiment nouns $(0.022)$.

Table 1. A summary of the sentiment of all of SentiWordNet cat-

\begin{tabular}{|l|l|l|l|}
\hline Part of Speech & Pos & Neg & Obj \\
\hline Adjectives & 0.106 & 0.151 & 0.743 \\
\hline Nouns & 0.022 & 0.034 & 0.944 \\
\hline Verbs & 0.026 & 0.034 & 0.940 \\
\hline Adverbs & 0.235 & 0.067 & 0.698 \\
\hline All & 0.043 & 0.054 & 0.903 \\
\hline
\end{tabular}

Nonetheless, it should be noted that SentiWordNet as an automatically created lexicon has a slightly biased performance on a corpus. More documents appear to be classified as positive rather than negative. Yet, the SentiWordNet approach yields a macro F1 measure of $56.8 \%$ and correctly classifies a similar percentage of all documents in the corpus (see Table 2).

Table 2. Experimental Results of SentiWordNet performance (Heerschop et al 2011)[6]

\begin{tabular}{|l|l|l|l|l|l|l|l|l|}
\hline & \multicolumn{3}{|l}{ Positive } & \multicolumn{1}{l|}{ Negative } & \multicolumn{3}{l|}{ Overall } \\
\hline Method & Precision & Recall & F1 & Precision & Recall & F1 & Accuracy & Macro \\
\hline SentiWordNet & $56.3 \%$ & $84.3 \%$ & $67.5 \%$ & $68.8 \%$ & $34.6 \%$ & $46.0 \%$ & $59.5 \%$ & $56.8 \%$ \\
\hline
\end{tabular}

In terms of accuracy, the SentiWordNet approach outperforms all other automated approaches such as WordNetRel, PageRankSeed, PageRankSWN.

\subsection{General Inquirer}

The Harvard General Inquirer (GI) is a lexicon attaching syntactic, semantic, and pragmatic information to partof-speech tagged words. General Inquirer includes 26 categories based on a variety of sources. The resource is mainly based on Harvard $I V-4$ categories. It is based on three semantic dimensions reflecting Charles Osgood's semantic differential findings regarding basic language universals: Positive-Negative, Strong-Weak, and ActivePassive. Two large valence categories are Posi$\boldsymbol{t i v}_{2}$ comprising 1,915 words of positive outlook, and Negativ (2,291 words of negative outlook). Separately go the words of pleasure, pain, virtue, and vice; words indicating overstatement and understatement, often reflecting presence or lack of emotional expressiveness; etc. Among other 
categories, General Enquirer encompasses three verb types:

1. IAV - 1,947 verbs giving an interpretative explanation of an action, such as encourage, mislead, flatter;

2. DAV - 540 straight descriptive verbs of an action or feature of an action, such as run, walk, write, read;

3. SV - 102 state verbs describing mental or emotional states, usually detached from specific observable events, such as "love, trust, abhor".

An instance of a GI entry is as follows: ADORE H4 Positiv Active Pleasur EMOT SV SUPV.

General Inquirer altogether comprises 677 verbs tagged as negative (e.g., abandon, abate, abdicate, abhor, abolish, abscond, abuse, accost, accuse, ache, admonish, adulterate, etc.) and 399 verbs tagged as positive ones (e.g., abide, abound, absolve, accede, accentuate, accept, accommodate, accomplish, accord, accrue, achieve, admit, etc.). Besides, additional sentiment tags have been attached to the verb items. For instance, abuse is tagged as negative strong, accomplish as positive strong; abandon, abdicate, abscond as negative weak and admit as positive weak. Abscond is also tagged as hostile. Such verbs as admire, adore, amuse, appeal, assure, calm, celebrate, cuddle, etc. belong to pleasure verbs. Pure emotive verbs tagged as EMOT encompass adore, amuse, assure, brood, depress, determine, detest, disappoint, dishearten, disturb, etc.

\subsection{MPQA Subjectivity Lexicon}

MPQA Subjectivity Lexicon provides a lexicon of 8,222 terms (labeled as subjective expressions), gathered from several sources. This lexicon contains a list of words, along with their POS-tagging, labelled with polarity (positive, negative, neutral) and intensity (strong, weak) [21].

$M P Q A$ comprises 1,318 verbs labelled as positive, negative, both or neutral. To be more precise, there are 377 positive verbs, 866 negative, 8 both and 67 neutral ones. All the verbs have been labelled according to their intensity as strong (855 items) and weak (463 items). For instance, strong subjectivity positive verbs are absove, accede, acclaim, admire, admit, adore, adulate, advocate, affirm, etc., strong subjectivity negative verbs are abase, abash, abhor, abominate, abscond, abuse, accuse, etc. The verbs brag, covet, demand, fawn, gloat, implore, lust, plead are labelled as both positive and negative strong subjectivity verbs. Of interest are strong subjectivity neutral items which in their majority are mental verbs: accentuate, air, allegorize, assess, believe, cogitate, comprehend, confide, conjecture, consider, contemplate, deduce, discern, evaluate, extemporize, feel, foretell, glean, imagine, imply, infer, intimate, know, metaphorize, need, philosophize, ponder, pray, proclaim, prognosticate, regard, scrutinize, soliloquize, suppose, surprise, theoretize, think, tint, view.

\subsection{Bing Liu's Opinion Lexicon}

Bing Liu's Opinion Lexicon focuses on polarity of lexical items. Altogether it comprises 1,072 negative opinion verbs (abolish, abominate, abort, abrade, abscond, abuse, accost, accurse, accuse, acerbate, ache, etc.) and 331 positive opinion verbs (abound, acclaim, accomplish, admire, adore, adulate, advance, advantage, advocate, affirm, etc.). Negative opinion verbs noticeably prevail over positive opinion verbs.

\subsection{Linguistic Inquiry and Word Count (LIWC)}

The development of $L I W C$ is considered to be a huge step towards the psychometrics of words and its authors claim that $L I W C$ can accurately identify emotion in language use. LIWC2007 software was first created by James W. Pennebaker et al. as a text analysis tool containing a special dictionary with word stored under particular categories. The labels in the dictionary were attached based on expert judges' ratings. The emotion word categories required human judges to evaluate which words were suited for which categories. For all subjective categories, an initial selection of word candidates was gleaned from dictionaries, thesauruses, questionnaires, and lists made by research assistants. Groups of three judges then independently rated whether each word candidate was appropriate to the overall word category. Among the multiple variables leveraged in the dictionary, there are posemo (positive emotion) and negamo (negative emotion) labels. The updated LIWC2015 dictionary dimension Affect Words comprises $3.67 \%$ of positive emotion words and $1.84 \%$ of negative emotion words in the whole dictionary of over 11,000 items. Negative emotion words include anxiety, anger and sadness words.

3. Discussion. On estimating the value of sentiment verbs in separate sentiment lexicons it is worthwhile comparing the agreement in labelling verbs (a) as sentiment (emotion bearing); (b) tagging the verbs as positive or negative across these lexicons. To this end Cohen's kappa statistic has been leveraged. The statistical analysis showed that there is little agreement in the content and structure of the analyzed lexicons. Table 3 features the basic kappa agreement values between the five sentiment lexicons.

Table 3. Cohen's kappa scores of agreement in the verb data across the lexicons

\begin{tabular}{|l|l|l|l|l|l|}
\hline Agreement & MPQA & Opinion & GI & LIWC & SentiWordNet \\
\hline MPQA & - & 0.12 & 0.04 & -0.57 & -0.16 \\
\hline Opinion & & - & -0.08 & -0.49 & -0.49 \\
\hline GI & & & - & -0.36 & -0.39 \\
\hline LIWC & & & & - & -0.53 \\
\hline SentiWordNet & & & & & - \\
\hline
\end{tabular}

It should be noted, however, that the lexicons do not disagree as much in labelling the verbs as negative or positive, as they differ in terms of their coverage and including different verbs. Overall, 5.4\% of all verbs found in all the five lexicons fully agree in the sentiment values attached to them. Noticeably, negative tags prevail over the positive ones. The highest agreement is observed between MPQA and Opinion Lexicon (0.12). LIWC and SentiWordNet feature the lowest agreement scores with any other lexicon (0.53). Table 4 presents the beginning of the combined list of the verbs in the alphabetical order which make up the core of verb sentiment vocabulary.

Table 4. The core of verb sentiment vocabulary (fragment)

$\begin{array}{ll}\text { abuse } & \text { neg } \\ \text { adore } & \text { pos } \\ \text { agonize } & \text { neg } \\ \text { alarm } & \text { neg } \\ \text { amuse } & \text { pos } \\ \text { anger } & \text { neg } \\ \text { attack } & \text { neg }\end{array}$

Discrepancies in the sentiment lexicons can be attributed to a number of factors, mainly, to varied methods of lexicons' compilation, different coverage, sentiment annotation techniques adopted, etc. All the disparities in the lexicons can be treated as close and distant periphery of sentiment 
verbs depending on the number of sentiment lexicons where the annotation overlaps.

On identifying the core list of the sentiment (emotive) verbs in English based on the sentiment lexicons, its data has been compared with other linguistic findings, namely, the list of words denoting emotions by Philip N. JohnsonLaird and Keith Oatley [7] and the list of words preveously compiled by the author based on traditional dictionaries and thsauri. The comparison revealed some minor inconsistencies stemming from the coverage of the resources under study.

4. Conclusion. Emotion words people use provide important psychological cues to their thought processes, emotional states, intentions, and motivations. The frequent usage of emotion words and especially strong intensity emotion words testifies to a high degree of immersion on the part of the speaker. The simultaneous development of highspeed personal computers, the Internet, and elegant new statistical strategies have helped usher in a new age of the psychological study of language [20, p.25). The existing sentiment lexicons used to conduct sentiment analysis of the texts is the manifestation of this trend.

The comparison of the sentiment lexicons under review in terms of their verb coverage and sentiment tagging revealed little agreement across the analyzed lexicons, though the core of the lexicons appears to be fairly stable. Only $5.4 \%$ of all the verbs found in the five lexicons fully agree in their sentiment value. One thing that can be inferred from the comparison of the lexicons is that negatively tagged verbs prevail over the positively tagged ones in all subjectivity lexicons. The findings reveal more verbs with negative meaning than the positive one. Cohen's kappa statistic leveraged to measure the agreement in labelling verbs across the lexicons. The highest agreement is observed between MPQA and Opinion Lexicon (0.12), whereas the lowest is between LIWC and SentiWordNet (-0.53). Mismatches in the verb coverage and annotation in the lexicons are mostly attributed to polysemy of the verbs and variety of methods of lexicons' creation.

The application of specific sentiment lexicons should take into consideration the purpose of a sentiment analysis. A more fine-grained classification of sentiment categories found in General Enquirer allows outlining a gamut of emotions such as pleasure, happiness, sadness, anger, etc. In addition, General Enquirer, MPQA Subjectivity Lexicon and SentiWordNet attach intensity labels and scores to the lexical items they comprise. Consequently, apart from the coverage and polarity reflected in the lexicons, granularity of distinctions can be advantageous for some NLP applications.

Despite the appeal of computerized language measures and available sentiment lexicons, they are still rather crude, often ignoring the polysemy of words. The findings suggest that modern stochastic models implemented in sentiment lexicons result in a tentative attempt to capture evasive connotative (emotive) meaning in the language system, exemplified here by the system of English verbs. Still, further explorations in this direction and constant upgrading of the resources look promising.
1. Ahire, Sagar. 2015. A Survey of sentiment lexicons. https://pdfs.semanticscholar.org

2. Baccianella, Stefano, Esuli, Andrea \& Fabrizio Sebastiani. 2010. SentiWordNet 3.0: An enhanced lexical resource for sentiment analysis and opinion mining. Proceedings of the International Conference on Language Resources and Evaluation, LREC 2010, 17-23 May 2010, Valletta, Malta. 2200-2204.

3. Bakhtin, Mikhail. M. 1996. The problem of speech genres. In Carol A. Emerson (ed.), Essays, speech genres \& other late essays, 60-102. Austin, TX: University of Texas Press.

4. Esuli, Andrea \& Fabrizio Sebastiani. 2006. SentiWordNet: A publicly available lexical resource for opinion mining. Proceesdings of the 5th Conference on Language Resources and Evaluation (LREC 2006), European Language Resources Association (ELRA). 417.

5. General Inquirer. http://www.wjh.harvard.edu/ inquirer/. (3 March, 2018).

6. Heerschop, Bas, Hogenboom, Alexander \& Flavius Frasincar. 2011. Sentiment lexicon creation from lexical resources. Proceedings of International Conference on Business Information Systems, 185-196 Springer.

7. Johnson-Laird, Philip N. \& Keith Oatley. 1989. The language of emotions: An analysis of a semantic field. Cognition and Emotion 3(2). 81-123.

8. Joshi, Aditya, Bhattacharyya, Pushpak \& Sagar Ahire. 2017. Sentiment resources: Lexicons and datasets. In Eric Cambia, Dipankar Das et al. (eds.), A Practical Guide to Sentiment Analysis, 85-106. Springer.

9. Klann-Delius, Gisela. 2015. Emotion in language. In Ulrike M. Lüdtke (ed.), Emotion in language:Theory - research - application.135-156. Amsterdam: John Benjamins.

10. Linguistic Inquirer and Word Count (LIWC). http://www.liwc.net/tryonline.php. (3 March, 2018.)

\section{REFERENCES}

11. Liu, Bing. 2012. Sentiment analysis and opinion mining, Morgan \& Claypool Publishers.

12. Louw, Bill. 2000. Contextual prosodic theory: Bringing semantic prosodies to life. In Chris Heffer, Helen Sauntson (eds.), Words in Context: A Tribute to John Sinclair on his Retirement, 48-94. English Language Research Discourse Analysis Monograph No. 18, Birmingham: University of Birmingham.

13. MPQA Subjectivity Lexicon.

http://mpqa.cs.pitt.edu/lexicons/subj_lexicon/. (3 March, 2018).

14. Opinion Lexicon. https://www.npmjs.com/package/opinionlexicon. (3 March, 2018).

15. Potts, Christopher. 2011. Sentiment Symposium Tutorial: Lexicons. San Francisco.

http://sentiment.christopherpotts.net/lexicons.html. (3 March, 2018).

16. SentiWordNet. http://sentiwordnet.isti.cnr.it/. (23 March, 2018).

17. Shakhovskij, Viktor. 1987. Kategorizacija emocij v leksikosemanticheskoj sisteme jazyka [Categorization of emotions in the lexico-semantic system of language]. Voronezh: Voronezh university.

18. Sinclair, John. 1996. The search for units of meaning. Textus IX. 75-106.

19. Stubbs, Michael. 1995. Collocations and semantic profiles: on the cause of the trouble with quantitative methods. Function of Language 2(1). 23-55.

20. Tausczik, Yla R. \& James W. Pennebaker. 2010. The psychological meaning of words: LIWC and computerized text analysis methods. Journal of Language and Social Psychology 29. 24-54.

21. Wiebe, Janyce, Wilson, Theresa \& Claire Cardie. 2005. Annotating expressions of opinions and emotions in language. Language Resources and Evaluation 39(2-3). 165-210. 